\title{
Genetic Predisposition to Allergic Rhinitis May Be a Causal Risk Factor for Bipolar Disorder and Major Depressive Disorder
}

\section{Wenjing Liao}

First Affiliated Hospital of Guangzhou Medical University

\section{Lijuan Song}

First Affiliated Hospital of Guangzhou Medical University Junyang Xie

First Affiliated Hospital of Guangzhou Medical University

\section{Gui Chen}

First Affiliated Hospital of Guangzhou Medical University

\section{Tianhao Liang}

First Affiliated Hospital of Guangzhou Medical University

\section{Ang Li}

First Affiliated Hospital of Guangzhou Medical University

Chunyi Zhang

First Affiliated Hospital of Guangzhou Medical University

\section{Meiqian Xu}

First Affiliated Hospital of Guangzhou Medical University Hao Li

First Affiliated Hospital of Guangzhou Medical University Jianlei Xie

First Affiliated Hospital of Guangzhou Medical University

\section{Pingchang Yang}

First Affiliated Hospital of Guangzhou Medical University

\section{Deming Han}

First Affiliated Hospital of Guangzhou Medical University

\section{Nanshan Zhong}

First Affiliated Hospital of Guangzhou Medical University

Xiaowen Zhang ( $\nabla$ entxiaowen@163.com )

First Affiliated Hospital of Guangzhou Medical University 
Keywords: Allergic rhinitis, Psychiatric disorders, Mendelian Randomization

Posted Date: March 7th, 2022

DOI: https://doi.org/10.21203/rs.3.rs-1400034/v1

License: (c) (i) This work is licensed under a Creative Commons Attribution 4.0 International License. Read Full License 


\section{Abstract}

There is increasing evidence suggest a link between allergic rhinitis (AR) and psychiatric disorders. However, casual associations between these variables remain uncertain. We performed bi-directional twosample Mendelian Randomization (MR) to examine the causality association of AR with 11 different psychiatric disorders or relevant traits. MR was conducted using the inverse-variance weighted method (IVW), MR-Egger and weighted median methods. Sensitivity analyses included the MR-Egger regression and MR pleiotropy residual sum and outlier test. AR from 2 different GWAS data was positively associated with bipolar disorder $(\mathrm{OR}=1.649,95 \% \mathrm{Cl}$ : 1.077-2.526; $\mathrm{P}=0.021 ; \mathrm{OR}=1.599 ; 95 \% \mathrm{Cl} 1.058-2.417$; $\mathrm{P}=0.026)$ and major depressive disorder $(\mathrm{OR}=1.539 ; 95 \% \mathrm{Cl} 1.007-2.353 ; \mathrm{P}=0.047)$, but not other psychiatric illnesses. Bidirectional analyses showed that bipolar disorder is negatively associated with AR (OR=0.964; 95\%Cl: 0.936-0.993; $P=0.015)$. There was no evidence for potential causal schizophrenia and effects of attention deficit/hyperactivity disorder on risk of AR by MR method, but MR pleiotropy residual outlier test suggested that attention deficit/hyperactivity disorder is negatively associated with AR after outlier correction (OR=0.976, 95\% Cl: $0.958-0.995, \mathrm{P}=0.012)$. This $\mathrm{MR}$ study indicated that $\mathrm{AR}$ was a causal risk factor for bipolar disorder and major depressive disorder, but not for other psychiatric disorders. Bipolar disorder and attention deficit/hyperactivity disorder may be a protective factor for AR. Further studies could be carried out to unravel the underlying mechanism in AR and psychiatric disorders.

\section{Introduction}

Allergic rhinitis (AR) is an inflammation in the nose caused by overreaction of the immune system to allergens in the air, affecting $10-40 \%$ of the general population. " "Allergic toxemia" was described as early as in 1930s, and manifest as a syndrome that include anxiety, fatigue, poor memory, irritability, and depression. ${ }^{2}$ Recently, a large body of evidence from prospective observational studies indicates that AR is a risk factor for several psychiatric disorders, such as insomnia, ${ }^{3}$ anxiety disorder, ${ }^{4}$ attention deficit/ hyperactivity disorder (ADHD), ${ }^{5}$ autism spectrum disorder (ASD), ${ }^{6}$ bipolar disorder, ${ }^{7}$ and major depressive disorder $(M D D)^{8}$. Nonetheless, these observational studies are unable to fully account for confounding factors and reverse causality, the causal nature of the association between AR and psychiatric disorders remains to be established.

Mendelian randomization (MR) is a novel epidemiological tool using genetic variants as instrumental variables (IVs) for investigating potential causal relationships between different human traits. ${ }^{9}$ Bidirectional MR analysis is an extension to the traditional MR approach, and could be used to evaluate whether an "exposure" causes the "outcome" and vice versa. The method has been popularized in modern literature, identifying important causal factors for various complex diseases.

Given the limited and inconsistent evidence in AR and psychiatric illnesses, current study used large-scale genome-wide association study (GWAS) data, and employed bidirectional MR to determine the potential cause-effect relationship between AR and 11 psychiatric disorders or relevant traits [insomnia, anorexia 
nervosa, anxiety disorder, panic disorder, post-traumatic stress disorder (PTSD), ADHD, ASD, bipolar disorder, MDD, obsessive-compulsive disorder (OCD) and schizophrenia].

\section{Methods}

\section{GWAS summary data}

This study is a bidirectional two-sample MR study using genetic instruments [single-nucleotide polymorphisms (SNPs)] predicting AR and psychiatric disorders from published GWAS studies and MR Base GWAS catalog (https://www.ebi.ac.uk/gwas/home). Only GWAS studies based on European populations were included in MR analysis to avoid genetic differences among populations. 9 GWAS studies associated with AR or relevant traits were from the UK Biobank (UKBB), FinnGen, and a metaanalysis of original GWAS studies. Supplementary Table $\mathbf{1} 1$ summarizes sample sizes, consortium and GWAS ID of allergic rhinitis GWASs included in Mendelian randomization. GWAS studies related to 11 psychiatric disorders were obtained from the Genetic Consortium for AN (GCAN), Psychiatric Genomics Consortium (PGC), FinnGen, UKBB, Bipolar Disorder Working Group of the Psychiatric Genomics Consortium, and Schizophrenia Working Group of the Psychiatric Genomics Consortium (Supplementary Table S2). ${ }^{10-12}$ We used a bidirectional design to assess the association of AR (Exposures) with psychiatric disorders (Outcomes) as well as to test whether psychiatric disorders (Exposures) cause AR(Outcomes).

\section{Assess instrument strength}

MR relies on three assumptions, i.e. the genetic predictors of exposure are strongly associated with the exposure of interest; the genetic predictors of exposure are independent from confounders of the exposure-outcome relation; and the genetic predictors are only linked to the outcome through affecting the exposure of interest (i.e. the exclusion-restriction assumption). ${ }^{13}$ To satisfy the first assumption, we only included SNPs with a genome-wide significant association with AR $\left(P<5^{\star} 10^{-8}\right) .{ }^{14}$ When the number of valid SNPS was greater than 3, F statistics was calculated to evaluate the strength of the associations between the IVs and AR. ${ }^{15} \mathrm{MR}$ analysis was allowed only when the $\mathrm{F}$ statistic was $>10$. In addition, we performed strict linkage disequilibrium (LD)-clumping restricted to $r^{2}<0.001$, Clumping distance $>$ $10000 \mathrm{~kb}$ to satisfy the independence of each SNP.

\section{Statistical analysis}

A random-effects inverse-variance weighted (IVW) method meta-analysis of the Wald ratio for individual SNPs was used to assess the cause-effect relationship between AR and the risk of psychiatric disorders. ${ }^{16}$ Weighted median and MR-Egger methods with different assumptions were also used. Weighted median method provides a causal estimate if at least $50 \%$ of the weight in the analysis comes from valid instrumental variables. ${ }^{17}$ MR-Egger method provides a valid effect estimate even if all SNPs are invalid instruments. Results are presented as odds ratios (OR) caused by each standard deviation (SD) increase or decrease in the AR and $95 \%$ confidence intervals (Cl). If the effect estimates were 
statistically significant $(P<0.05)$, power calculations were performed according to Brion et al. ${ }^{18} \mathrm{~A}$ value of power at $>80 \%$ indicates sufficient statistical power.

\section{Sensitivity analysis}

Since many SNPs have associations with multiple traits (pleiotropy), MR-Egger Regression ${ }^{19}$ and MR pleiotropy residual sum and outlier (MR-PRESSO) ${ }^{20}$ was performed. Deviation of the MR-Egger intercept from zero (at $\mathrm{p}<0.05)$ indicates horizontal pleiotropy or violation of the MR third assumption. MRPRESSO global test were performed to estimate for outlier, and where evident, using this method to correct the IVW-estimate by outlier removal where present.

The process of analyses was summarized in Fig. 1. All MR analyses were performed using $\mathrm{R}$ version (4.0.4) with the "TwoSampleMR" and "MRPRESSO" package.

\section{Results}

In this study, we investigated the causal relationships between AR, and psychiatric disorders by conducting bidirectional MR analysis. The full results are available in Supplementary Table S3.

\section{Effect of AR on psychiatric disorders}

After the IVs selection process, 7 of 9 GWAS studies related to AR were excluded due to the lack of SNPs with a genome-wide significance. These associated SNPs account for $0.4 \%$ of the variation in AR risk. The F statistic of this study were $12(\mathrm{~F}>10)$, which means the instruments used strongly predict the AR.

AR (ukb-a-447) had significant risk effects on bipolar disorder ( $\mathrm{OR}=1.649,95 \% \mathrm{Cl}$ : $1.077-2.526, \mathrm{P}=$ 0.021) (Table 1). Higher risk for bipolar disorder with AR was also apparent in weighted median method $(\mathrm{OR}=1.972,95 \% \mathrm{Cl}: 1.186-3.279, \mathrm{P}=0.009)$. However, MR-Egger method failed to establish an significant association, but the direction was consistent $(\mathrm{OR}=1.593,95 \% \mathrm{Cl}: 0.527-4.820, \mathrm{P}=0.412)$. Calculated based on 20,352 bipolar disorder cases and 31,358 controls in the outcome datasets with type 1 error at 0.05 demonstrated sufficient statistical power (0.96) to detect a causal effect of AR on bipolar disorder. MR-Egger regression (intercept $\beta=0.000, p=0.947$ ) failed to identify evidence to support the existence of unbalanced pleiotropy in the genetic instruments. The MR-PRESSO global test suggested the potential of outlier (rs118013485, rs1904522, and rs4580194) affecting AR with bipolar disorder as outcomes ( $\mathrm{p}<$ $\left.5^{\star} 10^{-4}\right)$. Following outlier correction, there was still evidence for a risk effect of AR on risk of bipolar disorder (Outlier-Corrected IVW OR = 1.510, 95\%Cl: 1.017-2.243, P = 0.041) (Supplementary Table S4). 
Table 1

Mendelian randomization analyses showing the effect estimates of allergic rhinitis and psychiatric disorders.

\begin{tabular}{|c|c|c|c|c|c|c|c|}
\hline $\begin{array}{l}\text { Exposure } \\
\text { (ID) }\end{array}$ & Outcome (ID) & nSNP & Method & $\begin{array}{l}\text { OR } \\
(95 \% \mathrm{Cl})\end{array}$ & $\begin{array}{l}P \\
\text { value }\end{array}$ & $\begin{array}{l}\text { Heterogeneity } \\
\text { P value }\end{array}$ & $\begin{array}{l}\text { Intercept } \\
\text { P value }\end{array}$ \\
\hline \multirow[t]{3}{*}{$\begin{array}{l}\text { Allergic } \\
\text { rhinitis(ukb- } \\
\text { a-447) }\end{array}$} & $\begin{array}{l}\text { Bipolar } \\
\text { disorder (ieu- } \\
\text { b-41) }\end{array}$ & 97 & IVW & $\begin{array}{l}1.649 \\
(1.077 \\
2.526)\end{array}$ & 0.021 & $5.40 \mathrm{E}-05$ & NA \\
\hline & & & $\begin{array}{l}\text { Weighted } \\
\text { median }\end{array}$ & $\begin{array}{l}1.972 \\
(1.186, \\
3.279)\end{array}$ & 0.009 & NA & NA \\
\hline & & & $\begin{array}{l}\text { MR } \\
\text { Egger }\end{array}$ & $\begin{array}{l}1.593 \\
(0.527, \\
4.820)\end{array}$ & 0.412 & 4.11E-05 & 0.947 \\
\hline \multirow[t]{3}{*}{$\begin{array}{l}\text { Allergic } \\
\text { rhinitis (ukb- } \\
\text { b-17241) }\end{array}$} & $\begin{array}{l}\text { Bipolar } \\
\text { disorder (ieu- } \\
\text { b-41) }\end{array}$ & 140 & IVW & $\begin{array}{l}1.599 \\
(1.058, \\
2.417)\end{array}$ & 0.026 & $1.22 \mathrm{E}-06$ & NA \\
\hline & & & $\begin{array}{l}\text { Weighted } \\
\text { median }\end{array}$ & $\begin{array}{l}1.668 \\
(0.993 \\
2.803)\end{array}$ & 0.053 & NA & NA \\
\hline & & & $\begin{array}{l}\text { MR } \\
\text { Egger }\end{array}$ & $\begin{array}{l}1.768 \\
(0.607, \\
5.155)\end{array}$ & 0.298 & $9.43 \mathrm{E}-07$ & 0.842 \\
\hline \multirow[t]{3}{*}{$\begin{array}{l}\text { Allergic } \\
\text { rhinitis (ukb- } \\
\text { b-17241) }\end{array}$} & $\begin{array}{l}\text { MDD (ebi-a- } \\
\text { GCST009981) }\end{array}$ & 143 & IVW & $\begin{array}{l}1.539 \\
(1.007 \\
2.353)\end{array}$ & 0.047 & 0.908 & NA \\
\hline & & & $\begin{array}{l}\text { Weighted } \\
\text { median }\end{array}$ & $\begin{array}{l}1.996 \\
(1.030, \\
3.868)\end{array}$ & 0.041 & NA & NA \\
\hline & & & $\begin{array}{l}\text { MR } \\
\text { Egger }\end{array}$ & $\begin{array}{l}1.362 \\
(0.611 \\
3.040)\end{array}$ & 0.451 & 0.899 & 0.726 \\
\hline \multirow[t]{3}{*}{$\begin{array}{l}\text { Bipolar } \\
\text { disorder(ieu- } \\
\text { b-41) }\end{array}$} & $\begin{array}{l}\text { Allergic } \\
\text { rhinitis(ukb-b- } \\
\text { 12753) }\end{array}$ & 14 & IVW & $\begin{array}{l}0.964 \\
(0.936 \\
0.993)\end{array}$ & 0.015 & 0.598 & NA \\
\hline & & & $\begin{array}{l}\text { Weighted } \\
\text { median }\end{array}$ & $\begin{array}{l}0.957 \\
(0.920, \\
0.995)\end{array}$ & 0.028 & NA & NA \\
\hline & & & $\begin{array}{l}\text { MR } \\
\text { Egger }\end{array}$ & $\begin{array}{l}1.149 \\
(0.957 \\
1.379)\end{array}$ & 0.162 & 0.822 & 0.285 \\
\hline $\begin{array}{l}\text { Note: Abbrev } \\
\text { polymorphis }\end{array}$ & $\begin{array}{l}\text { ons: MDD: M } \\
\text { OR: Odds ra }\end{array}$ & lepr & $\begin{array}{l}\text { e disord } \\
\text { ence int } \epsilon\end{array}$ & SNP: Ni & 0 & e nucleotide & \\
\hline
\end{tabular}


In another GWAS summary data, AR (ukb-b-17241) also had significant risk effects on bipolar disorder by IVW method (OR $=1.599,95 \% \mathrm{Cl}: 1.058-2.417, \mathrm{P}=0.026)$. Weighted median $(\mathrm{OR}=1.668,95 \% \mathrm{Cl}: 0.993-$ $2.803, P=0.053)$ and $M R$-Egger method $(O R=1.768,95 \% \mathrm{Cl}: 0.607-5.155, P=0.298)$ failed to demonstrate significant differences, but the association was consistent, with a similar OR (Table 1). The statistical power was $94 \%$, suggesting sufficient confidence to show a causal effect of AR on bipolar disorder. No evidence of pleiotropy was found by MR-Egger regression. However, the MR-PRESSO outlier test detected several specific horizontal pleiotropic outlier variants (rs1904522 and rs62385260). The association of AR with bipolar disorder was not statistically significant in MR-PRESSO outlier-corrected IVW analysis $(\mathrm{OR}=1.461,95 \% \mathrm{Cl} 0.971-2.199, \mathrm{P}=0.069)$ (Supplementary Table S4).

IVW method suggested significant risk effect of AR (ukb-b-17241) on MDD by IVW method (OR $=1.539$, $95 \% \mathrm{Cl}: 1.007-2.353, \mathrm{P}=0.047)$, but the weighted median $(\mathrm{OR}=1.996,95 \% \mathrm{C}: 0.968-4.119, \mathrm{P}=0.061)$ and MR-Egger $(\mathrm{OR}=1.362,95 \% \mathrm{Cl}: 0.611-3.040, \mathrm{P}=0.451)$ methods failed to confirm this association (Table 1). Moreover, not enough power $(0.79<0.80)$ to support a significant association between AR and MDD. No evidence of pleiotropy or outlier variants was found by either MR-Egger regression (intercept $\beta=$ $0.016, P=0.383)$ or MR-PRESSO outlier test $(P=0.914>0.05)$ (Supplementary Table S4).

No causal effects of AR on the risk of remaining psychiatric disorders were found by any of the three MR methods (Supplementary Table S3).

\section{Effect of psychiatric disorders on AR}

Based on the IVs selection process, anorexia nervosa, anxiety disorder, ASD, insomnia complaints, MDD, $O C D$, panic disorder, PTSD were excluded from further analysis due to insufficient number of SNPs with a genome-wide significance. $F$ statistics of 3 psychiatric disorders (ADHD, bipolar disorder, and schizophrenia) were 1271,383 , and 251 , respectively, indicating no weak instruments.

Bipolar disorder had significant protective effects on AR (ukb-b-12753) ( $\mathrm{OR}=0.964,95 \% \mathrm{Cl}: 0.936-0.993$, $\mathrm{P}=0.015)$ (Table 1$)$. Weighted median method yielded similar results $(\mathrm{OR}=0.957,95 \% \mathrm{Cl}: 0.920-0.995, \mathrm{P}$ $=0.028)$. but the MR-Egger method failed to determine an association $(\mathrm{OR}=1.149,95 \% \mathrm{Cl}: 0.957-1.379$, $P=0.162)$. The effect estimates of bipolar disorder on allergic rhinitis was $0.24(<80 \%)$ statistical power, indicating a high probability or false discoveries. No evidence of pleiotropy or outlier variants was found by either MR-Egger regression (intercept $\beta=-0.016, P=0.081$ ) or MR-PRESSO outlier test $(P=0.6185$ > 0.05).

There was no evidence for potential causal effects of ADHD, MDD, or schizophrenia on AR.

(Supplementary Table S3) The MR-PRESSO global test suggested the potential of outlier (rs112984125) affecting ADHD with AR (ukb-a-254, ukb-a-447) as outcomes $(p=0.009,0.008)$. Following outlier correction, there was evidence for a protective effect of ADHD on risk of AR (ukb-a-254: Outlier-Corrected IVW OR $=0.972,95 \% \mathrm{Cl}$ : 0.945-0.999, P = 0.041; ukb-a-447: Outlier-Corrected IVW OR $=0.984,95 \% \mathrm{Cl}$ : $0.973-0.995, P=0.006$ ) (Supplementary Table S4). Similarly, rs112984125, rs4916723, and rs 9677504 
affecting ADHD (ieu-a-1183) with AR (ukb-b-7178) as outcomes. There was evidence for a protective effect of ADHD on risk of AR (Outlier-Corrected IVW OR $=0.976,95 \% \mathrm{Cl}$ : $0.958-0.995, \mathrm{P}=0.012$ )

\section{(Supplementary Table S4).}

\section{Discussion}

AR and psychiatric disorders may share many physiological pathways, including inflammation and sleep disturbances. Inflammation plays an important role in psychiatric disorders beyond depression, deemed as a breakthrough finding in the last two decades. ${ }^{21}$ However, in the aspect of the neurophysiological mechanism, the possibility that psychiatric disorders may be related to nasal inflammatory processes involving the intranasal and the other pathways have never been considered. The contributions of nurture (i.e. the environment) and nature (i.e. genetics) have been long touted for their aetiological importance in both in AR and psychiatric disorders progression and clinical onset. Disappointingly, knowledge gains in these areas, while individually successful, have to a large extent occurred in isolation from each other.

In the present MR study, we systematically assessed the associations of AR with a broad range of psychiatric disorders. Our study demonstrated that genetically predicted AR was associated with an increased risk of bipolar disorder and MDD. We did not find any evidence of causal associations between $A R$ and the other psychiatric diseases. Bipolar disorder and ADHD may be a protective factor for AR but suffers from low power, we found no evidence of a causal association between the other psychiatric diseases and AR.

Bipolar disorder has a high heritability (approximately 70\%). There seems to be some common genetic risk with conditions such as Alzheimer's disease, diabetes and coronary heart disease. ${ }^{22}$ Hypertension (20.5\%), asthma (12.5\%) and hypothyroidism (8.1\%) were the top medical comorbidities found in bipolar patients. ${ }^{23}$ Specifically, asthma patients had higher rates of bipolar disorder than controls, and BD patients had higher rates of asthma than controls. ${ }^{24} \mathrm{~A}$ retrospective cohort study using the Taiwan National Health Insurance Research Database reported an increased risk ( $\mathrm{HR}=4.62,95 \% \mathrm{Cl} 3.17-6.75)$ of developing $B D$ in later life among those with AR. ${ }^{25}$ This bidirectional MR study determined a negative association between bipolar disorder and AR but had inadequate power, it showed that bipolar disorder may be a possible protective factor for AR. Several evidence has shown that patients with AR may develop bipolar disorder, Manalai et al ${ }^{26}$ reported that pollen-specific immunoglobulin E positivity was associated with worsening depression scores in bipolar disorder patients during high pollen season. Chen et $\mathrm{al}^{27}$ reported a significantly increased incidence of bipolar disorder in cases of AR (0.77 vs. 0.18 per 1000 person-years) and an increased risk ( $\mathrm{HR}=4.62,95 \% \mathrm{Cl} 3.17-6.75)$ of developing bipolar disorder in later life among those with AR. At present, there is no clinical and experimental data to support this conclusion. Therefore, our results should encourage further examine causality, epidemiology, mechanisms, and treatment potential for bipolar disorder on AR.

The cytokines including interleukin (IL)-1 $\beta$, IL- 6 and tumor-necrosis factor- $a$ (TNF- $\alpha$ ) play a role in the pathophysiology of both AR and bipolar disorders. ${ }^{28}$ However, a recent MR study showed that 20 
immunological proteins/traits (pro-inflammatory cytokines: IL-6, TNF-a, IL-12, IL-16, IL-17, IL-18; antiinflammatory cytokines: IL-1 receptor antagonist, IL-10, IL-13; chemokines: IL-8, monocyte chemoattractant protein-1; lymphoid growth-factors: soluble (s) IL-2Ra, IL-4, IL-7, IL-9; myeloid growth-factor: IL5; acute phase protein: C-Reactive Protein; immune cells: neutrophils, lymphocytes; neurotrophic factor: brain derived neurotrophic factor) were not associated with bipolar disorder. ${ }^{28}$ There is no doubt at all that the strongest and most consistent positive association were observed between AR and bipolar disorder in this MR study, further studies are warranted to explore these associations in a causal fashion.

AR is more prevalent in patients with MDD and those having family history of depressive disorder. ${ }^{29}$ The patients with AR had a 1.7-fold higher chance of being diagnosed with depression than did the controls. ${ }^{30}$ A meta-analysis showed that patients with AR had 1.6 times more chances to present depression compared to healthy controls. ${ }^{31}$ Thus, a close association between AR and MDD has been repeatedly identified by both observational and experimental studies. The present MR study provided new evidence that genetic predisposition to AR was associated with an increased risk of MDD. There is a major need of experimental and longitudinal investigations, as well as clinical trials to further our understanding of best practices in patients with comorbid AR and MDD and to find new therapeutic and preventative interventions.

ADHD is a severely impairing neurodevelopmental disorder affecting up to $7.1 \%$ in children and adolescents. ${ }^{32}$ Several epidemiological studies have reported that children with ADHD have a high risk of developing allergic diseases, but the explanation for the allergic disease manifestations remained ambiguous. ${ }^{32}$ A recently systematic review and meta-analysis confirmed that children with ADHD experienced $59 \%$ greater odds of having AR relative to the children without ADHD. The low statistical power most likely resulted from the statistical heterogeneity and the methodological diversity of the included studies, with a further potential confounder in factors that were not measured (e.g., climate, pollution or microbial agents). ${ }^{33}$ Some observation studies have reported no evidence of a link between allergy and ADHD. ${ }^{34}$ In recently MR study showed that there was little evidence for inferring a causal effect of ADHD on AR. ${ }^{35}$ We also confirmed there was no evidence for potential causal effects of ADHD, bipolar disorder, or schizophrenia on AR using the IVW method. Our MR-PRESSO analysis indicated the potential of outlier (rs112984125) affecting ADHD with AR (ukb-a-447) as outcomes $(p=0.0065)$. After the outlier correction, MR-PRESSO outlier test further supported the significant association with OR= $0.984,95 \% \mathrm{Cl}: 0.973-0.995, \mathrm{P}=0.006$. The results revealed that $\mathrm{ADHD}$ was a possible protective factor for AR. At present, there is no clinical and experimental data to support this conclusion. Therefore, our results should encourage further examine causality, mechanisms, and treatment potential for ADHD on AR.

In this study, we used a bidirectional design to assess the association of AR (Exposures) with psychiatric disorders (Outcomes) as well as to test whether psychiatric disorders (Exposures) causes AR(Outcomes). There are several strengths in this study. First, cause-effect relationship could not be possibly established by observational study, including meta-analysis from well-designed studies. MR analysis can work as an analogue to randomized controlled trial (RCT), likelihood of confounding effects and maximal avoidance 
of reverse causality. To our knowledge, this is the first study that investigated the potential bidirectional associations between the genetic liability for allergic rhinitis and psychiatric disorders using bidirectional MR. Second, population stratification bias was reduced in this study because SNP selection and summary-level data of psychiatric disorders were merely based on individuals of European descent and population structure was adjusted for in the GWASs. Moreover, we used all GWAS studies related to AR or psychiatric disorders for MR analysis, effectively avoiding selection bias. Among them, different AR related exposures were all significative risk factors for bipolar disorder, greatly increasing the reliability of this study.

Several limitations shall be noted in interpreting our study. First, we are unable to test for a comprehensive causal association between AR and psychiatric disorders, 4 of 11 psychiatric disorders summary statistics which we used as exposures were only available for significantly associated SNPs, the other psychiatric disorders including anorexia nervosa, anxiety disorder, ASD, insomnia complaints, OCD, panic disorder and PTSD were excluded from further analysis due to insufficient number of SNPS. Second, given bipolar I disorder and bipolar II disorder shared distinct characteristics, we were unable to examine the association between AR and different bipolar disorder phenotypes. ${ }^{36}$ Consequently, if our included genetic variants did not represent the risk of all subtypes of bipolar disorder, our obtained effect estimate was difficult to interpret. Third, bidirectional analyses could potentially detect dynastic effects. When findings suggested a potentially causal effect, possible bidirectional effects were further tested. There was no bidirectional causal relationship between AR and psychiatric disorders. Nevertheless, our study alone did not provide definitive evidence to absence of a causal association. Alternatively, there is possibility that the effect is nonlinear. Fourth, the summary level data that we used did not allow for stratified analyses by covariates of interest, such as age, sex, body mass index, smoking, and use of nasal hormone therapy. It is worth noting that AR is mainly determined by physical health and other environmental factors. Hence, it is inappropriate to deduce that genetic effects are independent of environmental factors.

\section{Conclusions}

AR, a treatable disease, is associated with bipolar disorder and possibly MDD but not with other psychiatric disorders. Further experimental investigations are needed to clarify whether treatment of AR may prevent the onset of bipolar disorders and MDD. Bipolar disorder and ADHD may be a protective factor for AR, the exact mechanism remains to be explained.

\section{Declarations}

\section{ACKNOWLEDGEMENTS}

We thank Dr Caisheng Li (State Key Laboratory of Respiratory Disease, National Clinical Research center for Respiratory Disease, Guangzhou Institute of Respiratory Disease, First Affiliated Hospital of Guangzhou Medical University, Guangzhou Medical University) and Dr Jihui Zhang(Li Chiu Kong Family 
Sleep Assessment Unit, Department of Psychiatry, Faculty of Medicine, The Chinese University of Hong Kong; Guangdong Mental Health Center, Guangdong Provincial People's Hospital, Guangdong Academy of Medical Sciences) for their assistance with proofreading the manuscript.

\section{CONTRIBUTORS}

Conception and design: W.J.L, L.J.S, J.Y.X, D.M.H, N.S.Z and X.W.Z; (II) Administrative support: X.W.Z; (III) Data analysis and interpretation: G.C, T.H.L, A.L, H.L, C.Y.Z, M.Q.X and J.L.X; (IV) Manuscript writing: W.J.L, L.J.S, P.C.Y and X.W.Z; (V) Final approval of manuscript: All authors.

\section{Data availability}

All data during this study are included in this published article and its Supplementary files.

\section{Funding}

This work was supported by National Key Clinical Specialty and Key medical discipline (2021-2023) project of Guangzhou.

\section{Declaration of interests}

We declare no competing interests.

\section{Ethical Considerations}

No ethical clearance was required for this research, for no patients were involved in the development of the research question or its outcome measures. Only secondary analysis was performed using published GWAS summary statistics available in the public domain.

\section{References}

1. Brozek JL, et al. Allergic Rhinitis and its Impact on Asthma (ARIA) guidelines-2016 revision. J Allergy Clin Immunol. 2017; 140: 950- 958.

2. Eisenberger Naomi I, Moieni Mona. Inflammation affects social experience: implications for mental health. World Psychiatry. 2020; 19: 109-110.

3. Liu J, Zhang X, Zhao Y, Wang Y. The association between allergic rhinitis and sleep: A systematic review and meta-analysis of observational studies. PLoS One. 2020; 15(2): e0228533.

4. Brew BK, et al. The familial aggregation of atopic diseases and depression or anxiety in children. Clin Exp Allergy. 2018; 48(6):703-711.

5. Guo MM, et al. Peanut Sensitivity and Allergic Rhinitis in Young Children are Associated with Attention-Deficit Hyperactivity Disorder Symptoms in Adolescence. Neuropsychiatr Dis Treat. 2020; 16:1349-1357. 
6. Dai YX, et al. Increased Risk of Atopic Diseases in the Siblings of Patients with Autism Spectrum Disorder: A Nationwide Population-Based Cohort Study. J Autism Dev Disord. 2019; 49(11):46264633 .

7. Kazour F, et al. Olfactory markers for depression: Differences between bipolar and unipolar patients. PLoS One. 2020; 15(8): e0237565.

8. Roxbury CR, et al. Association Between Rhinitis and Depression in United States Adults. J Allergy Clin Immunol Pract. 2019; 7(6):2013-2020.

9. Zheng J, et al. Recent Developments in Mendelian Randomization Studies. Curr Epidemiol Rep. 2017. 4(4):330-345.

10. Hammerschlag AR, et al. Genome-wide association analysis of insomnia complaints identifies risk genes and genetic overlap with psychiatric and metabolic traits. Nat Genet. 2017 Nov;49(11):15841592.

11. Coleman JRI, et al. Genome-wide gene-environment analyses of major depressive disorder and reported lifetime traumatic experiences in UK Biobank. Mol Psychiatry. 2020 Jul;25(7):1430-1446.

12. Li Z, et al. Genome-wide association analysis identifies 30 new susceptibility loci for schizophrenia. Nat Genet. 2017 Nov;49(11):1576-1583.

13. Davey Smith G, Hemani G. Mendelian randomization: genetic anchors for causal inference in epidemiological studies. Hum Mol Genet. 2014; 23(R1): R89-98.

14. Davies NM, Holmes MV, Davey Smith G. Reading Mendelian randomisation studies: a guide, glossary, and checklist for clinicians. BMJ. 2018; 362: k601.

15. Burgess S, Thompson SG; CRP CHD Genetics Collaboration. Avoiding bias from weak instruments in Mendelian randomization studies. Int J Epidemiol. 2011; 40(3):755-764.

16. Burgess S, Butterworth A, Thompson SG. Mendelian randomization analysis with multiple genetic variants using summarized data. Genet Epidemiol. 2013; 37(7):658-665.

17. Bowden J, Davey Smith G, Haycock PC, Burgess S. Consistent Estimation in Mendelian Randomization with Some Invalid Instruments Using a Weighted Median Estimator. Genet Epidemiol. 2016; 40(4):304-314.

18. Brion MJ, Shakhbazov K, Visscher PM. Calculating statistical power in Mendelian randomization studies. Int J Epidemiol. 2013; 42(5):1497-1501.

19. Bowden J, Davey Smith G, Burgess S. Mendelian randomization with invalid instruments: effect estimation and bias detection through Egger regression. Int J Epidemiol. 2015; 44(2):512-525.

20. Hemani G, et al. The MR-Base platform supports systematic causal inference across the human phenome. Elife. 2018.;7: e34408.

21. Miller AH. Beyond depression: the expanding role of inflammation in psychiatric disorders. World Psychiatry. 2020; 19(1):108-109.

22. Kerner B. Genetics of bipolar disorder. Appl. Clin. Genet. 2014;7:33-42. 
23. Patel RS, et al. Gender Differences and Comorbidities in U.S. Adults with Bipolar Disorder. Brain Sci. 2018 Sep 1;8(9):168.

24. Wu MK, et al. Significantly Higher Prevalence Rate of Asthma and Bipolar Disorder Co-Morbidity: A Meta-Analysis and Review Under PRISMA Guidelines. Medicine (Baltimore). 2016 Mar;95(13):e3217.

25. Chen $\mathrm{MH}$, et al. Risk of bipolar disorder among adolescents with allergic rhinitis: A nationwide longitudinal study. J Psychosom Res. 2015 Dec;79(6):533-6.

26. Manalai Partam, et al. Pollen-specific immunoglobulin E positivity is associated with worsening of depression scores in bipolar disorder patients during high pollen season. Bipolar Disord. 2012; 14 : 90-98.

27. Chen Mu-Hong, et al. Risk of bipolar disorder among adolescents with allergic rhinitis: A nationwide longitudinal study. J Psychosom Res. 2015; 79: 533-536.

28. Chen $\mathrm{MH}$, et al. Correlation of proinflammatory cytokines levels and reduced gray matter volumes between patients with bipolar disorder and unipolar depression. J Affect Disord. 2019 Feb 15;245:815.

29. Timonen M, et al. Atopy and depression: results from the Northern Finland 1966 Birth Cohort Study. Mol Psychiatry. 2003;8(8):738-44.

30. Cuffel B, Wamboldt M, Borish L, Kennedy S, Crystal-Peters J. Economic consequences of comorbid depression, anxiety, and allergic rhinitis. Psychosomatics. 1999 Nov-Dec;40(6):491-6.

31. Rodrigues $\mathrm{J}$, et al. Anxiety and depression risk in patients with allergic rhinitis: a systematic review and meta-analysis. Rhinology. 2021 Aug 1;59(4):360-373.

32. Thomas R, Sanders S, Doust J, Beller E, Glasziou P. Prevalence of attention-deficit/hyperactivity disorder: a systematic review and meta-analysis. Pediatrics. 2015 Apr;135(4):e994-1001.

33. Miyazaki $C$, et al. Allergic diseases in children with attention deficit hyperactivity disorder: a systematic review and meta-analysis. BMC Psychiatry. 2017 Mar 31;17(1):120.

34. Schmitt J, Buske-Kirschbaum A, Roessner V. Is atopic disease a risk factor for attentiondeficit/hyperactivity disorder? A systematic review. Allergy. 2010;65(12): 1506-1524.

35. Leppert B, et al. The Effect of Attention Deficit/Hyperactivity Disorder on Physical Health Outcomes: A 2-Sample Mendelian Randomization Study. Am J Epidemiol. 2021;190(6):1047-1055.

36. Carvalho AF, Firth J, Vieta E. Bipolar Disorder. N Engl J Med. 2020;383:58-66.

\section{Figures}


Genetic variants associated with allergic rhinitis or psychiatric disorders identified through published GWAS studies and GWAS catalog searching.

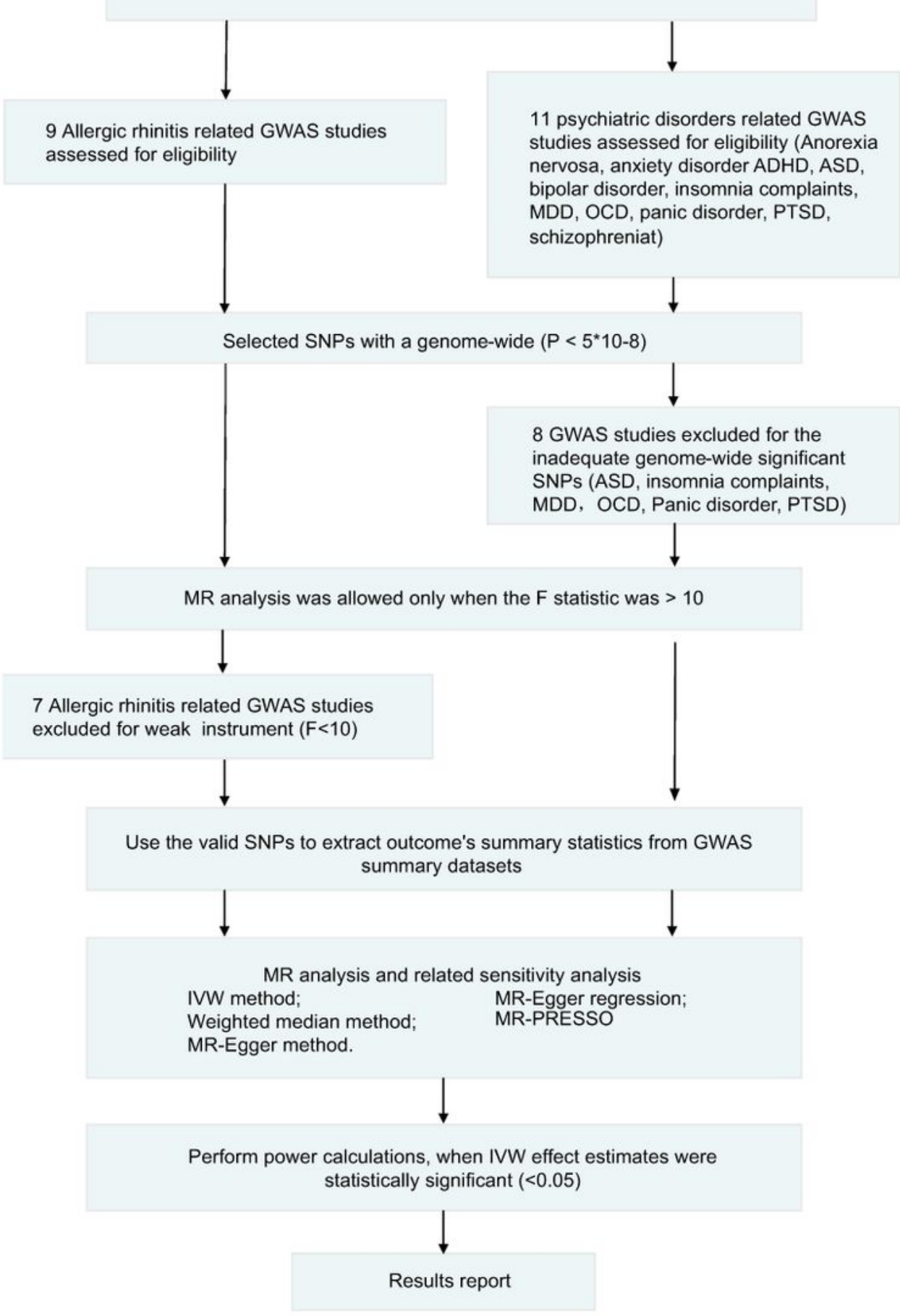

\section{Figure 1}

The analysis process of this study.

Note: Abbreviations: SNP: Single-nucleotide polymorphism; IVW: Inverse variance weighted method.

\section{Supplementary Files}


This is a list of supplementary files associated with this preprint. Click to download.

- SupplementTable.docx 